\title{
Predictors of advanced fibrosis in elderly patients with biopsy-confirmed nonalcoholic fatty liver disease: the GOASIA study
}

Panyavee Pitisuttithum', Wah Kheong Chan ${ }^{2}$, Panida Piyachaturawat ${ }^{1}$, Kento Imajo ${ }^{3}$, Atsushi Nakajima ${ }^{3}$, Yosuke Seki ${ }^{4}$, Kazunori Kasama ${ }^{4}$, Satoru Kakizaki ${ }^{5}$, Jian Gao Fan' ${ }^{6}$, Myeong Jun Song ${ }^{7}$, Seung Kew Yoon ${ }^{7}$, Yock Young Dan ${ }^{8}$, Laurentius Lesmana ${ }^{9}$, Khek Yu Ho ${ }^{8}$, Khean Lee Goh', Vincent Wai Sun Wong ${ }^{10}$ and Sombat Treeprasertsuk ${ }^{*}$

\begin{abstract}
Background: The Gut and Obesity in Asia (GOASIA) Workgroup was formed to study obesity and gastrointestinal diseases in the Asia Pacific region. We aimed to 1) compare the characteristics of elderly (i.e. age $\geq 60$ ) vs. nonelderly patients with biopsy-proven nonalcoholic fatty liver disease (NAFLD); 2) identify predictors of advanced fibrosis in elderly patients with NAFLD; and 3) assess the performance of non-invasive fibrosis scores in the prediction of advance fibrosis in the elderly population.
\end{abstract}

Methods: We abstracted the data of 1008 patients with NAFLD from nine centers across eight countries. Characteristics of elderly and non-elderly patients with NAFLD were compared using 1:3 sex-matched analysis.

Results: Of the 1008 patients, 175 were elderly [age 64 (62-67) years], who were matched with 525 non-elderly patients [46 (36-54) years]. Elderly patients were more likely to have advanced fibrosis (35.4\% vs. 13.3\%; $p<0.001$ ). By multivariable analysis, factors associated with advanced fibrosis in elderly patients included female sex [odds ratio (OR) 3.21; 95\% confidence interval (CI) 1.37-7.54] and hypertension (OR 3.68; 95\% Cl 1.11-12.23). The area under receiver-operating characteristics curve $(95 \% \mathrm{Cl})$ of aspartate aminotransferase-to-platelet ratio index, NAFLD fibrosis score and Fibrosis-4 index for predicting advanced fibrosis in elderly patients were 0.62 (0.52-0.72), 0.65 $(0.55-0.75)$ and $0.64(0.54-0.74)$ respectively.

Conclusions: Elderly patients with NAFLD had a higher prevalence of advanced fibrosis than non-elderly patients. Female and hypertension were predicting factors for advanced fibrosis in the elderly. Non-invasive fibrosis scores had a lower specificity in elderly.

Keywords: Advanced fibrosis, Elderly patients, Nonalcoholic fatty liver disease (NAFLD)

\footnotetext{
* Correspondence: battan5410@gmail.com; battan5410@yahoo.com

Part of the information has been submitted to the Digestive Disease Week ${ }^{\circledR}$

2017, held on May 6 - 9, 2017 at McCormick Place - Chicago, Illinois, United

States of America.

'Department of Medicine, Chulalongkorn University, Bangkok, Thailand

Full list of author information is available at the end of the article
}

C C The Author(s). 2020 Open Access This article is licensed under a Creative Commons Attribution 4.0 International License, which permits use, sharing, adaptation, distribution and reproduction in any medium or format, as long as you give appropriate credit to the original author(s) and the source, provide a link to the Creative Commons licence, and indicate if changes were made. The images or other third party material in this article are included in the article's Creative Commons licence, unless indicated otherwise in a credit line to the material. If material is not included in the article's Creative Commons licence and your intended use is not permitted by statutory regulation or exceeds the permitted use, you will need to obtain permission directly from the copyright holder. To view a copy of this licence, visit http://creativecommons.org/licenses/by/4.0/ The Creative Commons Public Domain Dedication waiver (http://creativecommons.org/publicdomain/zero/1.0/) applies to the data made available in this article, unless otherwise stated in a credit line to the data. 


\section{Background}

Nowadays, nonalcoholic fatty liver disease (NAFLD) is a growing problem and is becoming a major cause of chronic liver disease worldwide. NAFLD broadly encompasses two conditions with different prognosis: nonalcoholic fatty liver (NAFL) and nonalcoholic steatohepatitis (NASH). The prevalence of NASH has consistently been reported to increase with age besides being associated with obesity, diabetes mellitus and the metabolic syndrome [1-3]. Due to population aging, NAFLD is projected to increase globally.

Recently, Ballestri and colleagues conducted a comprehensive review of NAFLD studies and found age, sex and the metabolic syndrome to be major factors influencing NAFLD onset and progression. The incidence of NAFLD is higher in men $[4,5]$. In men, the prevalence of NAFLD increases during young adulthood to middle age and decline after the age of 5060 years [6,7]. In contrast, NAFLD is more common among elderly women with metabolic comorbidities [8]. In premenopausal women, the incidence of NAFLD is low but increases rapidly after menopause $[9,10]$. The striking gender difference has long been reported in NAFLD.

The global prevalence of NAFLD is about 25\% [11], while in Asia the disease has been reported to affect up to $30 \%$ of the general population in some studies [12-14]. However, data in the elderly population are limited. Currently, the proportion of older population is growing dramatically due to increased life expectancy and declining birth rates. Accordingly, the number of NAFLD patients would also inevitably increase. Predicting advanced fibrosis is necessary because of the higher risk of complications from chronic liver disease such as cirrhosis and hepatocellular carcinoma. In order to evaluate the liver disease severity, liver biopsy is gold standard. However, it is an invasive procedure and may be limited by sampling variability. So far there is no specific recommendation for elderly NAFLD in terms of diagnostic and fibrosis assessment $[15,16]$. Previous studies have shown that non-invasive fibrosis scores, including fibrosis-4 (FIB4) and NAFLD fibrosis score (NFS) have low specificity in elderly NAFLD patients [17].

The Gut and Obesity in Asia (GO ASIA) Workgroup was formed in November 2014 with the aim of studying relationship between obesity and gastrointestinal and liver diseases in the Asia Pacific region through multinational collaborative studies $[18,19]$. A database consisting of biopsy-proven NAFLD patients was formed through contribution from participating members of the GO ASIA Workgroup. We aimed to investigate factors associated with liver fibrosis in elderly (i.e. age $\geq 60$ ) vs. non-elderly patients with biopsy-proven NAFLD, and to identify predictors of advanced fibrosis in elderly patients. In addition, we evaluated the performance of non-invasive fibrosis scores in elderly NAFLD patients.

\section{Methods \\ Participating centers and cases}

This is a cross-sectional study of 1008 patients with biopsy-proven NAFLD in nine centers across eight countries in the GO ASIA Workgroup [19]. The centers that participated in this study and the duration of data collection were listed as follows: The Chinese University of Hong Kong, Hong Kong collected data from September 2006 to October 2015; University of Malaya, Malaysia collected data from November 2012 to October 2015; Chulalongkorn University, Thailand collected data from January 2007 to February 2014; Yotsuya Medical Cube / Gunma University Graduate School of Medicine, and Yokohama City University Graduate School of Medicine, Japan collected data from October 2009 to February 2016; Shanghai Jiaotong University School of Medicine, China collected data from June 2012 to December 2013; The Catholic University of Korea, Korea collected data from January 2009 to February 2016; National University of Singapore, Singapore collected data from October 2002 to October 2015; and Digestive Disease and Oncology GI Centre, Medistra Hospital, Indonesia collected data from May 2011 to September 2015. Ethical approval was obtained from each center prior to the commencement of the study. Verbal informed consent was obtained from all patients for being included in the study and the institutional review board approved the use of verbal consent.

NAFLD was diagnosed based on ultrasonography findings of fatty liver with exclusion of viral hepatitis $\mathrm{B}$ and $\mathrm{C}$ infection, significant alcohol intake, and the use of medications that can cause hepatic steatosis. Persistent elevated serum aminotransferase levels were the main indication for liver biopsy. Patients with other causes of chronic liver disease, incomplete histological data and without significant hepatic steatosis were excluded from the analyses. Demographic, anthropometric, clinical and laboratory data were collected using a standard protocol.

\section{Definitions}

Elderly patients were defined as age $\geq 60$ years. Obesity was defined as body mass index (BMI) $\geq 25.0 \mathrm{~kg} / \mathrm{m}^{2}$ [20]. Central obesity was defined as waist circumference $(\mathrm{WC})>90 \mathrm{~cm}$ for men and $>80 \mathrm{~cm}$ for women [21]. A patient was considered to have diabetes mellitus if there was a self-reported history of diabetes mellitus, or if fasting blood sugar was $\geq 7.0 \mathrm{mmol} / \mathrm{L}$. A patient was considered to have dyslipidemia if there was a self-reported history of dyslipidemia, if the 
serum total cholesterol (TC) was $\geq 5.2 \mathrm{mmol} / \mathrm{L}$, if the serum triglyceride (TG) was $\geq 1.7 \mathrm{mmol} / \mathrm{L}$, if the serum high-density lipoprotein (HDL) was $<1.0$ $\mathrm{mmol} / \mathrm{L}$ for men or $<1.3 \mathrm{mmol} / \mathrm{L}$ for women, or if the serum low-density lipoprotein (LDL) was $\geq 3.4 \mathrm{mmol} /$ L. All laboratory parameters included in the study were within 3 months before or after liver biopsy obtained. Hypertension was based on self-reported history. If they were using any drugs as evidence of the corresponding metabolic disorders also considered as having diabetes mellitus, dyslipidemia or hypertension.

\section{Non-invasive assessment of advanced fibrosis in patients with NAFLD}

The performance of the NAFLD fibrosis score (NFS), fibrosis 4 (FIB-4) score, and aspartate aminotransferase (AST) to platelet ratio index (APRI) in prediction of

Table 1 Characteristics of elderly and non-elderly NAFLD patients were compared using 1:3 sex-matched analysis

\begin{tabular}{|c|c|c|c|}
\hline Characteristics & Elderly NAFLD patients & Non-elderly NAFLD patients & $\boldsymbol{P}$ value \\
\hline median (IQR); n (\%) & $(n=175)$ & $(n=525)$ & \\
\hline Age (year) & $64(62-67)$ & $46(36-54)$ & $<0.001$ \\
\hline Female & $102(58.3 \%)$ & $306(58.3 \%)$ & 1 \\
\hline Weight (kg) & $69.45(62.08-78.13)$ & $78.5(68.1-97.3)$ & $<0.001$ \\
\hline Height (m) & $1.6(1.54-1.67)$ & $1.6(1.6-1.7)$ & $<0.001$ \\
\hline BMI $\left(\mathrm{kg} / \mathrm{m}^{2}\right)$ & $27.19(24.73-30.34)$ & $29.62(26.45-35)$ & $<0.001$ \\
\hline $\mathrm{BMI} \geq 25\left(\mathrm{~kg} / \mathrm{m}^{2}\right)$ & $126(72 \%)$ & $443(84.4 \%)$ & $<0.001$ \\
\hline Waist circumference $(\mathrm{cm})$ & 92.05 (81.75-99.63) & $91.2(78-102.5)$ & 0.813 \\
\hline Hip circumference $(\mathrm{cm})$ & $97(87.75-104)$ & $100(88-108)$ & 0.037 \\
\hline Central obesity & $126(85.1 \%)$ & $350(85.6 \%)$ & 0.987 \\
\hline Diabetes & $122(69.7 \%)$ & $237(45.1 \%)$ & $<0.001$ \\
\hline Hypertension & $122(74.8 \%)$ & $189(43.8 \%)$ & $<0.001$ \\
\hline Dyslipidemia & $148(84.6 \%)$ & $374(71.2 \%)$ & $<0.001$ \\
\hline Albumin (g/L) & $43(41-46)$ & $44(41-46)$ & 0.177 \\
\hline ALT (U/L) & $55(38-94)$ & $61(37-90)$ & 0.002 \\
\hline AST (U/L) & $43(26-66)$ & $40(29.8-68)$ & 0.327 \\
\hline $\operatorname{ALP}(\mathrm{U} / \mathrm{L})$ & $69(53.25-91)$ & $67(48.5-91.5)$ & 0.942 \\
\hline GGT (U/L) & $53(37-87)$ & $50(31-86)$ & 0.198 \\
\hline HbA1c (\%) & $6.65(5.9-7.33)$ & $6.1(5.6-7.2)$ & $<0.001$ \\
\hline Total cholesterol (mmol/L) & $4.4(3.5-5.33)$ & $5(4.3-5.7)$ & $<0.001$ \\
\hline LDL cholesterol (mmol/L) & $2.3(1.79-3.25)$ & $3(2.43-3.7)$ & $<0.001$ \\
\hline HDL cholesterol (mmol/L) & $1.25(1.1-1.44)$ & $1.2(1.01-1.5)$ & 0.248 \\
\hline Triglycerides (mmol/L) & $1.4(1.1-1.8)$ & $1.4(1.1-1.9)$ & 0.509 \\
\hline Hemoglobin $(g / d L)$ & $14.1(13.4-15.08)$ & $13.9(13.1-15.1)$ & 0.775 \\
\hline Platelet $\left(10^{9} / \mathrm{L}\right)$ & $227.5(177.5-280.75)$ & $251(202-297)$ & $<0.001$ \\
\hline WBC $\left(10^{9} / \mathrm{L}\right)$ & $6.57(5.6-8)$ & $7.3(5.9-8.8)$ & 0.015 \\
\hline NAFLD Activity Score (NAS) & $4(3-5)$ & $4(3-5)$ & 0.332 \\
\hline NAS $\geq 5$ & $58(33.1 \%)$ & $221(40.2 \%)$ & 0.097 \\
\hline NASH & $118(67.4 \%)$ & $335(63.8 \%)$ & 0.386 \\
\hline Significant fibrosis ( $\geq F 2$ ): histopathology & $86(49.1 \%)$ & $138(26.3 \%)$ & $<0.001$ \\
\hline Advanced fibrosis ( $\geq F 3$ ): histopathology & $62(35.4 \%)$ & $70(13.3 \%)$ & $<0.001$ \\
\hline Cirrhosis & $27(15.4 \%)$ & $25(4.8 \%)$ & $<0.001$ \\
\hline NAFLD fibrosis score (NFS) ${ }^{a}$ & $-0.75(1.49)$ & $-1.91(1.88)$ & $<0.001$ \\
\hline APRI score ${ }^{a}$ & $1.18(2.02)$ & $0.74(1.55)$ & 0.02 \\
\hline FIB-4 score ${ }^{a}$ & $3.88(6.72)$ & $1.58(3.93)$ & $<0.001$ \\
\hline
\end{tabular}


advanced fibrosis in the elderly and non-elderly NAFLD patients were assessed.

\section{Histological data}

Histopathological findings were reported according to the Non-Alcoholic Steatohepatitis Clinical Research Network Scoring System [22]. NAFLD Activity Score (NAS) was defined as the un-weighted sum of the scores for steatosis which was the presence of significant hepatic steatosis ( $>5 \%$ of hepatocytes) (0-3), lobular inflammation $(0-3)$, and ballooning $(0-2)$; thus ranging from 0 to 8. NASH was defined as steatosis with hepatocyte ballooning and inflammation with/without fibrosis [23].

The slides were review by pathologist in each center. Fibrosis stages 3 and 4 were considered as advanced fibrosis (F3-4).

\section{Statistical analysis}

We performed 1:3 matching by sex for elderly and nonelderly NAFLD patients. Continuous variables were reported as mean, standard deviation and analyzed using t-test. For non-normal distribution data were reported as median, interquartile range and analyzed using Mann-Whitney U test. Categorical variables were reported as percentages and analyzed using chi-square test. Factors associated with advanced fibrosis in elderly group were identified using binary logistic regression analysis. Multivariable analysis was performed by including all variables that had $p$-values $<0.1$ from univariable analysis. Area under receiver-operating characteristics curve (AUROC) and its 95\% confidence intervals were generated to determine performance of fibrosis prediction scores. A two-tailed $P$-value $<0.05$ was considered statistically significant. Analyses were performed using SPSS 22.0 (SPSS Inc., Chicago, Illinois, U.S.).

\section{Results}

Characteristics of elderly and non-elderly NAFLD patients

Of 1008 patients with biopsy-proven NAFLD, 175 patients were elderly, who were matched with 525 nonelderly patients. The percentages of elderly NAFLD patients from each country included in the study are shown in supplemental table 1. The median (IQR) age of elderly NAFLD and non-elderly NAFLD patients were 64 (62-67) and $46(36-54)$ years. In the elderly group, $102(58.3 \%)$ patients were female. Characteristics of elderly and non-elderly NAFLD patients in different centers are shown in supplemental table 2.

The elderly NAFLD patients were more likely to have diabetes mellitus, hypertension and dyslipidemia than non-elderly patients. Elderly NAFLD patients had significantly lower BMI [27.19 (24.73-30.34) vs 29.62 $\left.(26.45-35) \mathrm{kg} / \mathrm{m}^{2}, p<0.001\right]$ and hip circumference [97 $(87.75-104)$ vs $100(88-108) \mathrm{cm}, p=0.037]$. Elderly NAFLD patients had lower alanine aminotransferase [55 (38-94) vs $61(37-90) \mathrm{U} / \mathrm{L}, p=0.002]$, platelet count $\left[227.5(177.5-280.75)\right.$ vs $251(202-297) \times 10^{9} / \mathrm{L}$, $p<0.001]$, white blood count [6.57 (5.6-8) vs $7.3(5.9-8.8)$ $\left.\times 10^{3} / \mathrm{mm}^{3}, p=0.015\right]$, total cholesterol [4.4 (3.5-5.33) vs 5 (4.3-5.7) $\mathrm{mmol} / \mathrm{L}, p<0.001]$ and LDL cholesterol [2.3 $(1.79-3.25)$ vs $3(2.43-3.7) \mathrm{mmol} / \mathrm{L}, \mathrm{p}<0.001]$ than nonelderly NAFLD patients. Elderly patients had significantly

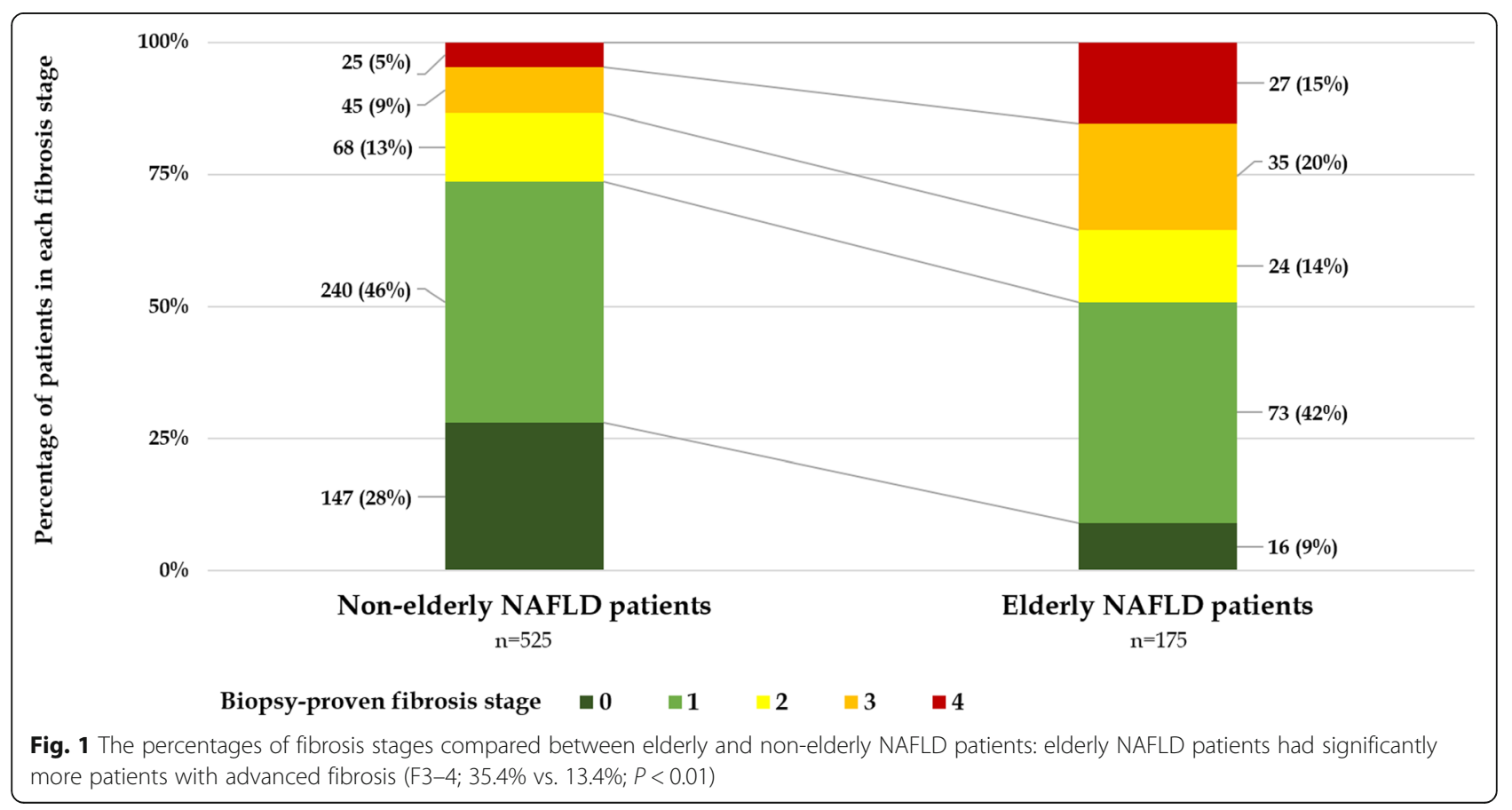


higher hemoglobin A1c than non-elderly patients [6.65 $(5.9-7.33)$ vs $6.1(5.6-7.2) \%, p<0.001$ ] (Table 1$)$.

For the pathology findings, NASH was not difference between elderly and non-elderly NAFLD patients $(67.4 \%$ vs.63.8\%, $p=0.386$ ). Elderly NAFLD patients were more likely to have advanced fibrosis (F3-4; $35.4 \%$ vs. 13.3\%, $p<0.001)$ and cirrhosis $(15.4 \%$ vs. $4.8 \%, \mathrm{p}<0.001)$ than non-elderly NAFLD patients (Fig. 1). Elderly patients also had higher fibrosis scores: APRI $(1.18 \pm 2.02$ vs. $0.74 \pm 1.55, p=0.02)$, NFS $(-0.75 \pm 1.49$ vs. $-1.91 \pm 1.88$, $\mathrm{p}<0.001)$, and FIB- 4 index $(3.88 \pm 6.72$ vs. $1.58 \pm 3.935$, $\mathrm{p}<0.001$ ) (Table 1).

\section{Characteristics of elderly NAFLD patients with and without advanced fibrosis}

Focusing on the elderly NAFLD patients, over one-third of them had advanced fibrosis (35.4\%) including stage 3 fibrosis (F3; 20\%) and stage 4 fibrosis (F4; 15.4\%). Elderly NAFLD patients with advanced fibrosis were more likely to be female $(72.6 \%$ vs. $50.4 \% ; p=0.005)$, obesity $(83.9 \%$ vs $65.5 \% ; p=0.01)$, central obesity $(94.7 \%$ vs. $79.1 \% ; p=$ $0.009)$, diabetes (79\% vs $64.6 \% ; p=0.047)$ and hypertension $(88.3 \%$ vs. $67 \% ; p=0.002)$ (Table 2$)$.

By univariate analysis, female sex, obesity, central obesity, diabetes and hypertension were associated

Table 2 Characteristics of elderly groups with advanced fibrosis (F3-4) vs. non-advanced fibrosis (F0-2)

\begin{tabular}{|c|c|c|c|}
\hline Elderly NAFLD & Non-advanced fibrosis & Advanced fibrosis & $\begin{array}{l}P \text { - } \\
\text { value }\end{array}$ \\
\hline Characteristics, median (IQR); n (\%) & $(\boldsymbol{n}=113)$ & $(\boldsymbol{n}=62)$ & \\
\hline Age (year) & $64(62-66)$ & $65(62-68)$ & 0.118 \\
\hline Female & $57(50.4 \%)$ & $45(72.6 \%)$ & 0.005 \\
\hline Weight (kg) & $68.35(59.88-78.00)$ & $70.7(64-79)$ & 0.565 \\
\hline Height (m) & $1.6(1.57-1.70)$ & $1.6(1.54-1.68)$ & 0.227 \\
\hline BMI $\left(\mathrm{kg} / \mathrm{m}^{2}\right)$ & $26.68(23.94-29.12)$ & $27.55(25.89-31.22)$ & 0.055 \\
\hline $\mathrm{BMI} \geq 25\left(\mathrm{~kg} / \mathrm{m}^{2}\right)$ & $74(65.5 \%)$ & $52(83.9 \%)$ & 0.01 \\
\hline Waist circumference $(\mathrm{cm})$ & $92.05(84-100)$ & $96(89.50-102.50)$ & 0.017 \\
\hline Hip circumference $(\mathrm{cm})$ & $98(92.00-104.63)$ & $101(95.50-109)$ & 0.158 \\
\hline Central obesity & $72(79.1 \%)$ & $54(94.7 \%)$ & 0.009 \\
\hline Diabetes & $73(64.6 \%)$ & $49(79.0 \%)$ & 0.047 \\
\hline Hypertension & $69(67 \%)$ & $53(88.3 \%)$ & 0.002 \\
\hline Dyslipidemia & $94(83.2 \%)$ & $54(87.1 \%)$ & 0.493 \\
\hline Albumin (g/L) & $43(42-45)$ & $43(40-46)$ & 0.637 \\
\hline ALT (U/L) & $52.5(37-89.75)$ & $66(36-91)$ & 0.276 \\
\hline AST (U/L) & $38.5(27.25-56.75)$ & $51(34-82)$ & 0.086 \\
\hline ALP (U/L) & $63(49-117.25)$ & $69.5(69-70)$ & 0.615 \\
\hline GGT (U/L) & $48(34.25-70.75)$ & $75(42.75-122.5)$ & 0.002 \\
\hline HbA1c (\%) & $6.5(5.9-7.4)$ & $6.7(6.13-7.28)$ & 0.435 \\
\hline Total cholesterol (mmol/L) & $4.4(3.5-5.38)$ & $4.35(3.53-5.1)$ & 0.483 \\
\hline LDL cholesterol (mmol/L) & $2.32(1.8-3.23)$ & $2.23(1.7-3.08)$ & 0.389 \\
\hline HDL cholesterol (mmol/L) & $1.2(1.03-1.4)$ & $1.3(1.1-1.5)$ & 0.068 \\
\hline Triglycerides (mmol/L) & $1.4(1.2-1.79)$ & $1.5(1.13-1.8)$ & 0.833 \\
\hline Hemoglobin $(g / d L)$ & $14.1(13.4-14.8)$ & $13.45(12.63-14.68)$ & 0.055 \\
\hline Platelet $\left(10^{9} / \mathrm{L}\right)$ & $229.5(193-284.25)$ & $205(145.75-243.25)$ & 0.003 \\
\hline WBC $\left(10^{9} / L\right)$ & $7(5.8-8.23)$ & $6.9(5.83-8.15)$ & 0.638 \\
\hline NAFLD Activity Score (NAS) & $4(3-5)$ & $4(4-5)$ & 0.023 \\
\hline NAS $\geq 5$ & $32(28.3 \%)$ & $26(41.9 \%)$ & 0.067 \\
\hline Platelet count $\leq 140 \times 10^{9} / \mathrm{L}$ & $10(8.8 \%)$ & $13(21 \%)$ & 0.023 \\
\hline NAFL fibrosis score (NFS) & $-1.00(1.48)$ & $-0.24(1.4)$ & 0.005 \\
\hline APRI score ${ }^{a}$ & $0.91(1.41)$ & $1.74(2.85)$ & 0.077 \\
\hline FIB-4 score ${ }^{a}$ & $3.06(5.15)$ & $5.57(9.01)$ & 0.095 \\
\hline
\end{tabular}

$A P R I$ AST to platelet ratio index, $B M I$ body mass index; Non-Alcoholic Steatohepatitis (NAS) score ${ }^{a}$ mean (SD) 
with advanced fibrosis among the elderly NAFLD patients. By multivariable analysis, factors associated with advanced fibrosis in elderly patients included female sex (OR 3.21; 95\%CI 1.37-7.54) and hypertension (OR 3.68; 95\%CI 1.11-12.23). In contrast, diabetes (OR 3.38; 95\%CI 1.70-6.73) and platelet count less than $140 \times 10^{9} / \mathrm{L}$ (OR 4.18 ; 95\%CI $1.32-$ 13.25) were factors associated with advanced fibrosis in non-elderly patients (Table 3 ).

\section{The performance of non-invasive fibrosis scores in elderly NAFLD patients}

Box plot of non-invasive fibrosis scores in each liver biopsy-proven fibrosis stage is shown in Fig. 2.The AUROCs of APRI, NFS and FIB-4 to predict advanced fibrosis in the elderly NAFLD patients were as follows: 0.62 $(0.52-0.72) p=0.03,0.65(0.55-0.75) p=0.01$, and 0.64 $(0.54-0.74) \mathrm{p}=0.01$, respectively (Table 4 ). Comparison of the AUROCs among APRI, NFS, and FIB-4 showed no significant difference within elderly NAFLD patients (APRI vs NFS, $p=0.551$, APRI vs FIB- $4, p=0.308$ and NFS vs FIB-4, $p=0.828$ ). The flow diagram evaluating the performance of non-invasive fibrosis scores in elderly NAFLD patients is shown in supplemental fig. 1 . Comparison between percentage of advanced fibrosis in elderly NAFLD patients and non-elderly NAFLD patients from each non-invasive fibrosis score is shown in supplemental table 3. Sensitivity, specificity, positive predictive value (PPV) and negative predictive value (NPV) of APRI, NFS and FIB-4 in both elderly and non-elderly NAFLD are shown in supplemental table 4.

\section{Discussion}

This multicenter study on 700 biopsy-proven NAFLD patients revealed that a significant proportion of elderly patients had advanced fibrosis (35.4\%). Liver is an organ with age and sex-related changes [24], and the complications from chronic liver disease increase with age, contributing significantly to health care burden. Besides, we demonstrated that the non-invasive scores were higher in elderly NAFLD patients than non-elderly patients. To better understand how to hinder disease progression, reliable baseline data are needed to identify predictors for early intervention and close monitoring. In this study, we found that elderly NAFLD patients with advanced fibrosis were more likely to be female and were more likely to have hypertension.

An earlier study from Noureddin et al. compared between elderly and non-elderly patients with NAFLD showed the predominance of NAFLD in elderly female patients. Their elderly NAFLD patients were more likely to be hypertensive, had lower mean BMI and smaller waist circumference. Moreover, They had a higher mean AST/ ALT ratio, lower mean platelet count, and higher mean APRI score that were suggested as indices of more advanced liver disease [3]. Similar to the previous study, our elderly NAFLD patients had lesser mean BMI and hip circumference. This group of patients was more likely to have hypertension, diabetes mellitus, and dyslipidemia. It is worth noting that we must be aware that the elderly group may have NAFLD even if they have low BMI.

To identify patients who have advanced fibrosis, liver biopsy is the gold standard, but this procedure has some limitations due to its invasive nature and the potential complications. Non-invasive fibrosis scores, for example, APRI, NFS and FIB-4 score were developed to predict liver fibrosis. However, the validation of these scores were included only in the minority of elderly group [25] or some studies did not include any subjects with ages $\geq 65$ years [26, 27]. Meta-analysis in 2016, most of patients aged less than 60, showed FIB-4 score had better diagnostic accuracy than NFS and BARD score. In nonelderly NAFLD, our study also supported that FIB-4 had better diagnostic accuracy than NFS and APRI [28].

Table 3 Univariate analysis and multivariate analysis for predictors of advanced fibrosis in the elderly and non-elderly patients with NAFLD

\begin{tabular}{|c|c|c|c|c|c|c|c|c|}
\hline \multirow[t]{3}{*}{ Characteristics } & \multicolumn{4}{|c|}{ Elderly NAFLD patients } & \multicolumn{4}{|c|}{ Non-elderly NAFLD patients } \\
\hline & \multicolumn{2}{|l|}{ Univariate analysis } & \multicolumn{2}{|l|}{ Multivariate analysis } & \multicolumn{2}{|l|}{ Univariate analysis } & \multicolumn{2}{|c|}{ Multivariate analysis } \\
\hline & $\mathrm{OR}(95 \% \mathrm{Cl})$ & P-value & OR $(95 \% \mathrm{Cl})$ & P-value & OR $(95 \% \mathrm{Cl})$ & P-value & OR (95\%Cl) & P-value \\
\hline Age $\geq 70$ years & $2.22(0.85-5.80)$ & 0.103 & $2.24(0.43-11.61)$ & 0.338 & & & & \\
\hline Female & $2.60(1.33-5.08)$ & 0.005 & $3.21(1.37-7.54)$ & 0.007 & $0.89(0.53-1.47)$ & 0.639 & $0.59(0.3-1.15)$ & 0.119 \\
\hline $\mathrm{BMI} \geq 25 \mathrm{~kg} / \mathrm{m}^{2}$ & $2.74(1.25-6.00)$ & 0.011 & $2.41(0.85-6.8)$ & 0.098 & $1.13(0.55-2.31)$ & 0.741 & $0.94(0.35-2.54)$ & 0.904 \\
\hline Central obesity & $4.75(1.34-16.88)$ & 0.016 & $4.09(0.68-24.67)$ & 0.124 & $1.24(0.53-2.88)$ & 0.62 & $1.18(0.44-3.18)$ & 0.747 \\
\hline Diabetes mellitus & $2.07(1.00-4.26)$ & 0.049 & $2.29(0.88-5.93)$ & 0.088 & $3.88(2.22-6.79)$ & $<0.001$ & $3.38(1.7-6.73)$ & 0.001 \\
\hline Hypertension & $3.73(1.53-9.07)$ & 0.004 & $3.68(1.11-12.23)$ & 0.034 & $1.29(0.76-2.21)$ & 0.346 & $1.59(0.8-3.12$ & 0.183 \\
\hline Dyslipidemia & $1.36(0.56-3.33)$ & 0.494 & & & $1.01(0.58-1.76)$ & 0.97 & & \\
\hline Platelet count $\leq 140 \times 10^{9} / \mathrm{L}$ & $2.73(1.12-6.67)$ & 0.027 & $2.43(0.59-9.91)$ & 0.217 & $4.06(1.85-8.88)$ & $<0.001$ & $4.18(1.32-13.25)$ & 0.015 \\
\hline
\end{tabular}




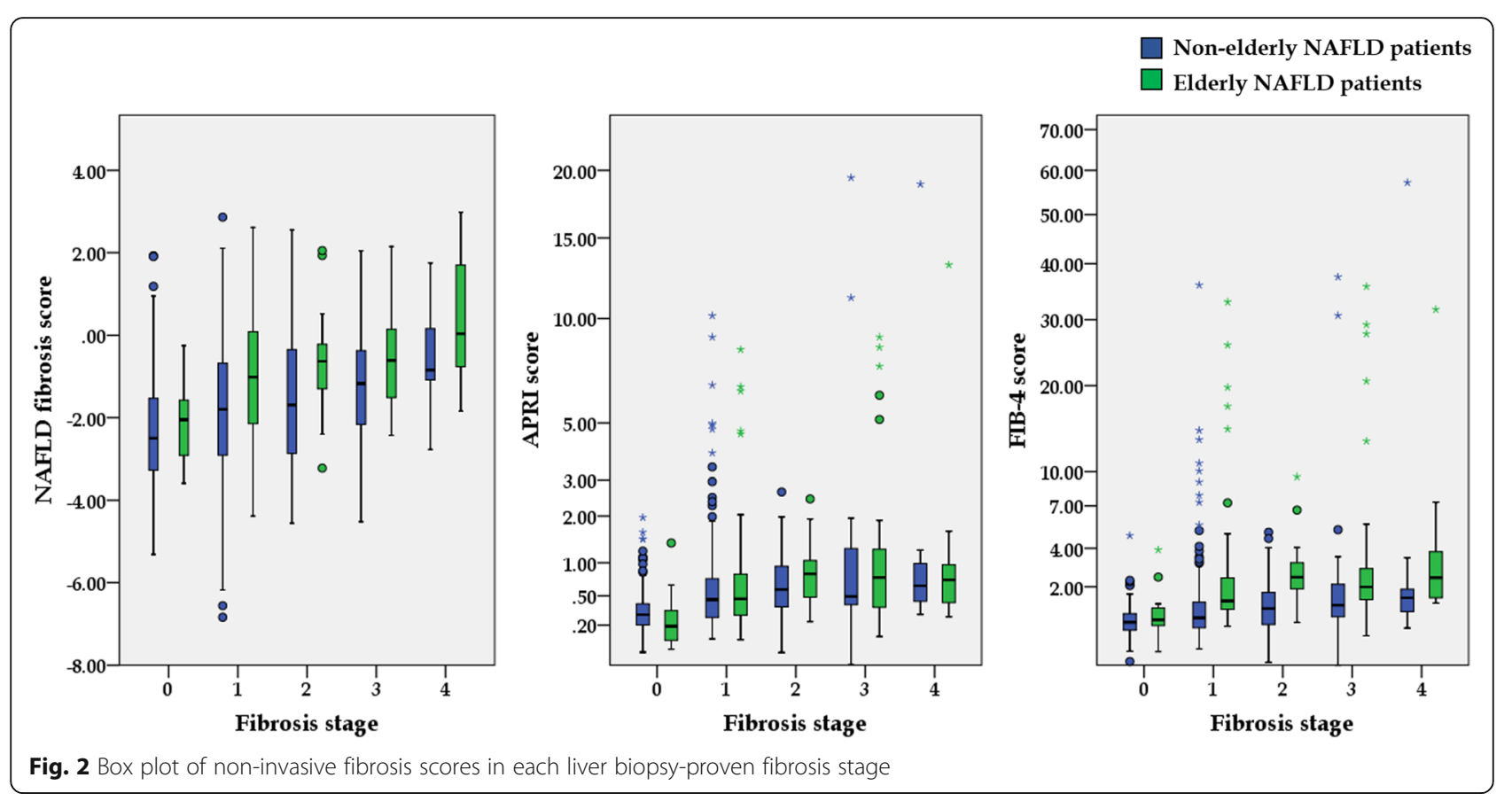

Recently, McPherson et al. reported that the NAFLD fibrosis score and FIB-4 had low specificity for advanced fibrosis in patients aged $\geq 65$ years leading to a high false positive rate. New cutoffs have been proposed to improve the accuracy of the NFS and FIB-4 score in patients ages $\geq 65$ years [17]. From our data, we found that the NFS and FIB-4 had a lower specificity when using lower cutoffs. When validating by using the new cutoffs by McPherson et al., specificity was increased in elderly, however, sensitivity was low (supplemental table 5).

Aging promotes metabolic disturbances causing an increase in lipid accumulation in non-adipose tissues, including the liver. With reduced physical activity in aging, insulin resistance and hyperinsulinemia promote obesity and the onset of the metabolic syndrome. NASH is considered to be a manifestation of metabolic syndrome $[29,30]$. In the present study, the prevalence of advanced liver fibrosis was high in elderly patients, particularly in women with central obesity. The gender difference of the metabolic disturbance is most evident in postmenopausal women. Although the mechanisms of menopause-induced metabolic dysfunction are largely unknown, estrogen depletion is thought to responsible for the underlying pathological mechanism [31]. This observation requires further study to confirm.

The strength of this study includes the use of a wellcharacterized cohort of biopsy-proven NAFLD patients from multiple centers in Asia. The proportion of elderly patients in the present study is greater than those reported in previous studies $[3,4]$. One of the limitations may arise from the liver biopsy results and its assessment which was reported by the local pathologists of each center and they were not reviewed centrally by a panel of pathologists. However, interobserver agreement for advanced fibrosis tends to be better, and our study focused on advanced fibrosis. Secondly, the sampling errors of liver biopsy and observer variability may affect the pathological results interpretation [22]. Lastly, the study could be affected by

Table 4 The performance of AST to Platelet Ratio Index (APRI), NAFLD fibrosis score (NFS) and fibrosis 4 (FIB-4) in prediction of advanced fibrosis in elderly NAFLD patients and non-elderly NAFLD patients

\begin{tabular}{llll}
\hline Area under the curve $(95 \% \mathrm{Cl})$ & APRI & NFS & FIB-4 \\
\hline Non-elderly NAFLD & $0.67(0.60-0.74) p<0.001$ & $0.68(0.61-0.75) p<0.001$ & $0.71(0.64-0.78) p<0.001$ \\
& $(n=480)$ & $(n=475)$ & $(n=480)$ \\
Elderly NAFLD & $0.62(0.52-0.72) p=0.03$ & $0.65(0.55-0.75) p=0.01$ & $0.64(0.54-0.74) p=0.01$ \\
& $(n=132)$ & $(n=131)$ & $(n=132)$ \\
All patients & $0.66(0.61-0.72) p<0.001$ & $0.70(0.65-0.75) p<0.001$ & $0.73(0.67-0.78) p<0.001$ \\
& $(n=612)$ & $(n=606)$ & $(n=612)$ \\
\hline
\end{tabular}


selection bias as the sample came from tertiary centers and specialist liver clinics, thus the findings may not be applicable to general clinic setting.

\section{Conclusions}

Elderly patients with NAFLD had a higher prevalence of advanced fibrosis than non-elderly patients. Female sex and hypertension were predictive factors for the presence of advanced fibrosis in elderly. We should be aware of this in our aging society. Performance of fibrosis prediction by using non-invasive scoring system including APRI, NFS and FIB-4 were not good enough to predict the presence of advanced fibrosis stage in the elderly.

\section{Supplementary information}

Supplementary information accompanies this paper at https://doi.org/10. 1186/s12876-020-01240-z.

Additional file 1: Supplemental fig. 1. The flow diagram evaluating the performance of non-invasive fibrosis scores in elderly and non-elderly NAFLD patients. Supplemental table 1. The percentages of elderly NAFLD patients $(n=175)$ and non-elderly NAFLD patients from each country included in the study (total $=1008$ ). Supplemental table 2. Characteristics of elderly and non-elderly NAFLD patients in different centers. Supplemental table 3. Comparison between percentages of advanced fibrosis in elderly and non-elderly NAFLD patients.

Supplemental table 4. Thesensitivity, specificity, positive predictive value (PPV) and negative predictive value (NPV) of APRI, NFS and FIB-4 in both elderly and non-elderly NAFLD patients. Supplemental table $\mathbf{5}$. AUROC, sensitivity, specificity, positive predictive value (PPV) and negative predictive value (NPV) of APRI, NFS and FIB-4 in elderly NAFLD patients when elderly defined as $\geq 65$ according to McPherson et al.

\section{Abbreviations}

APRI: Aspartate aminotransferase-to-platelet ratio index; AUROC: Area under receiver-operating characteristics curve; FIB-4: Fibrosis 4 score; NAFLD: Nonalcoholic fatty liver disease; NAS: NAFLD activity score; NFS: NAFLD fibrosis score; NPV: Negative predictive value; PPV: Positive predictive value

\section{Acknowledgments}

We would like to thank the supporting team of the Gut and Obesity in Asia (GOASIA) Workgroup for database. Additionally, we would like to thank the Gl research coordinator and statistician, Gl Division, King Chulalongkorn Memorial hospital, Miss Kanokwan Sornsiri and Miss Chonlada Phathong.

\section{Authors' contributions}

ST and PP1 designed the study. PP1, WC, PP2, KI, AN, YS, KK, SK, JF, MS, SY, $Y D, L L, K H, K G, V W, S T$ contributed to data acquisition. ST and PP1 analyzed and interpreted the data. PP1, PP2 and ST drafted the manuscript. ST, WC and $\mathrm{W}$ revised the manuscript critically for important intellectual content. All authors read and approved the final manuscript.

\section{Funding}

The grant support organization including the Liver Research Unit of the Faculty of Medicine, Chulalongkorn University, as well as the funding from Fatty Liver Research Unit, Division of Gastroenterology, Chulalongkorn University. The funders had no role in study design, decision to publish and preparation of the manuscript.

\section{Availability of data and materials}

The datasets used and/or analyzed during the current study are available from the corresponding author on reasonable request.

\section{Ethics approval and consent to participate}

Ethics approval was obtained from each center prior to the commencement of the study. The Joint Chinese University of Hong Kong - New Territories East Cluster Clinical Research Ethics Committee, China; Medical Ethics Committee of University Malaya Medical Center, Malaysia; the Institutional Review Board of the Faculty of Medicine, Chulalongkorn University, Thailand; the Institutional Review Boards of Yotsuya Medical Cube and Gunma University, Japan; the Ethics Committee of Yokohama City University, Japan; Ethics Committees of Xinhua Hospital affiliated with the Shanghai Jiao Tong University School of Medicine, China; the Ethics Review Board of the Catholic University of Korea, Korea; the National Healthcare Group Domain Specific Review, Singapore; the Medistra hospital's local ethics committee, Indonesia approved this study. This study was conducted in accordance with the Declaration of Helsinki. As retrospective study, our center recommended to use verbal consent, verbal informed consent was obtained from all patients for being included in the study and the institutional review board approved the use of verbal consent.

\section{Consent for publication}

Not applicable.

\section{Competing interests}

The authors declare that they have no competing interests.

\section{Author details}

${ }^{1}$ Department of Medicine, Chulalongkorn University, Bangkok, Thailand. ${ }^{2}$ Department of Medicine, University of Malaya, Kuala Lumpur, Malaysia. ${ }^{3}$ Department of Gastroenterology and Hepatology, Yokohama City University Graduate School of Medicine, Yokohama, Japan. ${ }^{4}$ Weight Loss and Metabolic Surgery Centre, Yotsuya Medical Cube, Tokyo, Japan. ${ }^{5}$ Department of Gastroenterology and Hepatology, Gunma University Graduate School of Medicine, Gunma, Japan. ${ }^{6}$ Department of Gastroenterology, Shanghai Jiaotong University School of Medicine, Shanghai, China. ${ }^{7}$ Department of Internal Medicine, The Catholic University of Korea, Seoul, Korea.

${ }^{8}$ Department of Medicine, National University of Singapore, Singapore, Singapore. 'Digestive Disease and Oncology Gl Centre, Medistra Hospital, Jakarta, Indonesia. ${ }^{10}$ Department of Medicine and Therapeutics, The Chinese University of Hong Kong, Hong Kong, China.

Received: 25 July 2019 Accepted: 26 March 2020

Published online: 06 April 2020

\section{References}

1. Benedict $M$, Zhang $X$. Non-alcoholic fatty liver disease: an expanded review. World J Hepatol. 2017;9(16):715-32.

2. Sayiner M, Koenig A, Henry L, Younossi ZM. Epidemiology of nonalcoholic fatty liver disease and nonalcoholic Steatohepatitis in the United States and the rest of the world. Clin Liver Dis. 2016;20(2):205-14.

3. Noureddin M, Yates KP, Vaughn IA, Neuschwander-Tetri BA, Sanyal AJ, McCullough A, et al. Clinical and histological determinants of nonalcoholic steatohepatitis and advanced fibrosis in elderly patients. Hepatology. 2013; 58(5):1644-54

4. Ballestri S, Nascimbeni F, Baldelli E, Marrazzo A, Romagnoli D, Lonardo A. NAFLD as a sexual dimorphic disease: role of gender and reproductive status in the development and progression of nonalcoholic fatty liver disease and inherent cardiovascular risk. Adv Ther. 2017:34(6):1291-326.

5. Xu C, Yu C, Ma H, Xu L, Miao M, Li Y. Prevalence and risk factors for the development of nonalcoholic fatty liver disease in a nonobese Chinese population: the Zhejiang Zhenhai study. Am J Gastroenterol. 2013;108(8): 1299-304.

6. Eguchi Y, Hyogo H, Ono M, Mizuta T, Ono N, Fujimoto K, et al. Prevalence and associated metabolic factors of nonalcoholic fatty liver disease in the general population from 2009 to 2010 in Japan: a multicenter large retrospective study. J Gastroenterol. 2012;47(5):586-95.

7. Kojima S, Watanabe N, Numata M, Ogawa T, Matsuzaki S. Increase in the prevalence of fatty liver in Japan over the past 12 years: analysis of clinical background. J Gastroenterol. 2003;38(10):954-61.

8. Ludwig J, Viggiano TR, McGill DB, Oh BJ. Nonalcoholic steatohepatitis: Mayo Clinic experiences with a hitherto unnamed disease. Mayo Clin Proc. 1980 55(7):434-8 
9. Florentino GS, Cotrim HP, Vilar CP, Florentino AV, Guimaraes GM, Barreto VS. Nonalcoholic fatty liver disease in menopausal women. Arq Gastroenterol. 2013;50(3):180-5

10. Hamaguchi M, Takeda N, Kojima T, Ohbora A, Kato T, Sarui H, et al. Identification of individuals with non-alcoholic fatty liver disease by the diagnostic criteria for the metabolic syndrome. World J Gastroenterol. 2012; 18(13):1508-16.

11. Bertolotti M, Lonardo A, Mussi C, Baldelli E, Pellegrini E, Ballestri S, et al. Nonalcoholic fatty liver disease and aging: epidemiology to management. World J Gastroenterol. 2014;20(39):14185-204.

12. Younossi ZM, Koenig AB, Abdelatif D, Fazel Y, Henry L, Wymer M. Global epidemiology of nonalcoholic fatty liver disease-meta-analytic assessment of prevalence, incidence, and outcomes. Hepatology. 2016;64(1):73-84.

13. Fan JG, Kim SU, Wong WW. New trends on obesity and NAFLD in Asia. J Hepatol. 2017:67(4):862-73.

14. Chitturi S, Wong WW, Farrell G. Nonalcoholic fatty liver in Asia: firmly entrenched and rapidly gaining ground. J Gastroenterol Hepatol. 2011; 26(Suppl 1):163-72.

15. Nascimbeni F, Pais R, Bellentani S, Day CP, Ratziu V, Loria $P$, et al. From NAFLD in clinical practice to answers from guidelines. J Hepatol. 2013;59(4): 859-71.

16. Chalasani N, Younossi Z, Lavine JE, Charlton M, Cusi K, Rinella M, et al. The diagnosis and management of nonalcoholic fatty liver disease: practice guidance from the American Association for the Study of Liver Diseases. Hepatology. 2018;67(1):328-57.

17. McPherson S, Hardy T, Dufour JF, Petta S, Romero-Gomez M, Allison M, et al Age as a confounding factor for the accurate non-invasive diagnosis of advanced NAFLD fibrosis. Am J Gastroenterol. 2017;112(5):740-51.

18. Koh JC, Loo WM, Goh KL, Sugano K, Chan WK, Chiu WY, et al. Asian consensus on the relationship between obesity and gastrointestinal and liver diseases. J Gastroenterol Hepatol. 2016;31(8):1405-13.

19. Chan WK, Treeprasertsuk S, Imajo K, Nakajima A, Seki Y, Kasama K, et al. Clinical features and treatment of nonalcoholic fatty liver disease across the Asia Pacific region-the GO ASIA initiative. Aliment Pharmacol Ther. 2018; 47(6):816-25.

20. Anuurad E, Shiwaku K, Nogi A, Kitajima K, Enkhmaa B, Shimono K, et al. The new $B M I$ criteria for asians by the regional office for the western pacific region of $\mathrm{WHO}$ are suitable for screening of overweight to prevent metabolic syndrome in elder Japanese workers. J Occup Health. 2003;45(6): 335-43.

21. Alberti KG, Zimmet P, Shaw J, Group IDFETFC. The metabolic syndrome--a new worldwide definition. Lancet. 2005;366(9491):1059-62.

22. Kleiner DE, Brunt EM, Van Natta M, Behling C, Contos MJ, Cummings OW, et al. Design and validation of a histological scoring system for nonalcoholic fatty liver disease. Hepatology. 2005;41(6):1313-21.

23. Yeh MM, Brunt EM. Pathological features of fatty liver disease. Gastroenterology. 2014;147(4):754-64

24. Cvitanovic Tomas T, Moskon M, Mraz M, Rozman D. Computational Modelling of liver metabolism and its applications in research and the clinics. Acta Chim Slov. 2018;65(2):253-65.

25. Angulo P, Hui JM, Marchesini G, Bugianesi E, George J, Farrell GC, et al. The NAFLD fibrosis score: a noninvasive system that identifies liver fibrosis in patients with NAFLD. Hepatology. 2007;45(4):846-54.

26. Kruger FC, Daniels CR, Kidd M, Swart G, Brundyn K, van Rensburg C, et al. APRI: a simple bedside marker for advanced fibrosis that can avoid liver biopsy in patients with NAFLD/NASH. S Afr Med J. 2011;101(7):477-80.

27. Shah AG, Lydecker A, Murray K, Tetri BN, Contos MJ, Sanyal AJ, et al. Comparison of noninvasive markers of fibrosis in patients with nonalcoholic fatty liver disease. Clin Gastroenterol Hepatol. 2009;7(10):1104-12.

28. Sun W, Cui H, Li N, Wei Y, Lai S, Yang Y, et al. Comparison of FIB-4 index NAFLD fibrosis score and BARD score for prediction of advanced fibrosis in adult patients with non-alcoholic fatty liver disease: a meta-analysis study. Hepatol Res. 2016;46(9):862-70.

29. Amarapurkar D, Kamani P, Patel N, Gupte P, Kumar P, Agal S, et al. Prevalence of non-alcoholic fatty liver disease: population based study. Ann Hepatol. 2007;6(3):161-3
30. Park SH, Jeon WK, Kim SH, Kim HJ, Park DI, Cho YK, et al. Prevalence and risk factors of non-alcoholic fatty liver disease among Korean adults. J Gastroenterol Hepatol. 2006;21(1 Pt 1):138-43.

31. Quinn MA, Xu X, Ronfani M, Cidlowski JA. Estrogen deficiency promotes hepatic Steatosis via a glucocorticoid receptor-dependent mechanism in mice. Cell Rep. 2018;22(10):2690-701.

\section{Publisher's Note}

Springer Nature remains neutral with regard to jurisdictional claims in published maps and institutional affiliations.
Ready to submit your research? Choose BMC and benefit from:

- fast, convenient online submission

- thorough peer review by experienced researchers in your field

- rapid publication on acceptance

- support for research data, including large and complex data types

- gold Open Access which fosters wider collaboration and increased citations

- maximum visibility for your research: over $100 \mathrm{M}$ website views per year

At BMC, research is always in progress.

Learn more biomedcentral.com/submissions 